\title{
Information Leakage Attacks on Emerging Non-Volatile Memory and Countermeasures
}

\author{
Mohammad Nasim Imtiaz Khan \\ School of Electrical Engineering and Computer Science \\ The Pennsylvania State University \\ University Park, Pennsylvania \\ muk392@psu.edu
}

\author{
Swaroop Ghosh \\ School of Electrical Engineering and Computer Science \\ The Pennsylvania State University \\ University Park, Pennsylvania \\ szg212@psu.edu
}

\begin{abstract}
Emerging Non-Volatile Memories (NVMs) suffer from high and asymmetric read/write current and long write latency which can result in supply noise, such as supply voltage droop and ground bounce. The magnitude of supply noise depends on the old data and the new data that is being written (for a write operation) or on the stored data (for a read operation). Therefore, victim's write operation creates a supply noise which propagates to adversary's memory space. The adversary can detect victim's write initiation and can leverage faster read latency (compared to write) to further sense the Hamming Weight (HW) of the victim's write data by detecting read failures in his memory space. These attacks are specifically possible if exhaustive testing of the memory for all patterns, all possible location combinations, all possible parallel read/write conditions are not performed under bit-to-bit process variations and specified $\left(-10^{\circ} \mathrm{C}\right.$ to $\left.90^{\circ} \mathrm{C}\right)$ and unspecified temperature ranges (i.e., less than $-10^{\circ} \mathrm{C}$ and greater than $\left.90^{\circ} \mathrm{C}\right)$. Simulation result indicates that adversary can sense HW of victim's (near-by) write data = $66.77 \%$, and further narrow the range based on read/write failure characteristics. Side Channel Attacks can utilize this information to strengthen the attacks.
\end{abstract}

\section{CCS CONCEPTS}

- Security and privacy $\rightarrow$ Hardware attacks and countermeasures; $\bullet$ Hardware $\rightarrow$ Memory and dense storage;

\section{KEYWORDS}

Emerging NVM, RRAM, Security, Information Leakage, Hamming Weight, Side Channel Attack

\section{ACM Reference Format:}

Mohammad Nasim Imtiaz Khan and Swaroop Ghosh. 2018. Information Leakage Attacks on Emerging Non-Volatile Memory and Countermeasures. In Proceedings of ISLPED '18: International Symposium on Low Power Electronics and Design (ISLPED '18). ACM, New York, NY, USA, 6 pages. https://doi.org/10.1145/3218603.3218649

Permission to make digital or hard copies of all or part of this work for personal or classroom use is granted without fee provided that copies are not made or distributed for profit or commercial advantage and that copies bear this notice and the full citation on the first page. Copyrights for components of this work owned by others than ACM must be honored. Abstracting with credit is permitted. To copy otherwise, or republish, to post on servers or to redistribute to lists, requires prior specific permission and/or a fee. Request permissions from permissions@acm.org.

ISLPED '18, July 23-25, 2018, Seattle, WA, USA

(C) 2018 Association for Computing Machinery.

ACM ISBN 978-1-4503-5704-3/18/07.

https://doi.org/10.1145/3218603.3218649

\section{INTRODUCTION}

Emerging Non-Volatile Memory (NVM) technologies, such as SpinTransfer Torque RAM (STTRAM), Magnetic RAM (MRAM), Resistive RAM (RRAM), Phase Change Memory (PCM) and Ferroelectric RAM (FRAM) have drawn significant attention due to low static-power operation, high density, high speed and the inherent non-volatility [1-5]. These memories are proposed for different applications. For example, STTRAM can replace SRAM in L2/L3 cache $[6,7]$ due to compatible speed and endurance. Both STTRAM and RRAM are proposed to replace eFlash [6, 8]. PCM-based Solid State Drive (SSD), namely Optane is already marketed by Intel [9]. Furthermore, NVM enables low-power computations and novel architectures $[6,7,10]$. In addition to storage, NVMs are also investigated for novel applications, such as neuromorphic computing, ambient sensor, security primitive etc. [6,10]. Although promising, unique characteristics of NVMs introduce new threats to data security. Therefore, it is necessary to investigate their vulnerabilities before their mass adoption.

Vulnerabilities of emerging NVMs: Most of the emerging NVMs are susceptible to ambient parameters, such as temperature and magnetic field which can be used to launch Denial-of-Service (DoS) attacks [11, 12]. In [13], it has been pointed out that NVMs suffer from asymmetric and high read/write current (i.e., read/write current for data ' 0 ' and data ' 1 ' are different) which can be exploited to launch Side-Channel Attack (SCA) [14]. NVMs also suffer from supply voltage droop [15]. The ground bounce phenomena and the role of supply noise (i.e. droop and ground bounce) have also been explored in [16]. It has been shown that adversary can write a specific data pattern in his memory space which generates a deterministic supply noise in emerging NVM-based Last Level Cache (LLC). This noise can propagate to victim's memory space and affect his read/write operation causing DoS and fault injection attacks [16].

The root cause of supply noise is high write current and long write latency. Extremely high current $(\sim 50 \mathrm{~mA}$ assuming $100 \mu \mathrm{A} / \mathrm{bit})$ is drawn from the supply for a full cache line (512bit) write operation. This creates two issues:

(a) Supply voltage droop: On-chip voltage regulator or power supply keeps the supply voltage constant. However, the supply voltage, $\mathrm{V}_{\mathrm{dd}}$ (distributed in metal $\mathrm{M}_{9}$ ) reaches the memory bitcell (implemented in metal $M_{1}$ ) via a power-grid RC network (Fig. 1). The interconnect resistance creates a significant voltage droop at the bitcell due to the high current drawn during read/write operation (Fig. 1). Voltage droop reduces voltage headroom for the bitcell and increases the write latency (for write) and decreases the sense margin (for read). It can eventually lead to read/write failure. 


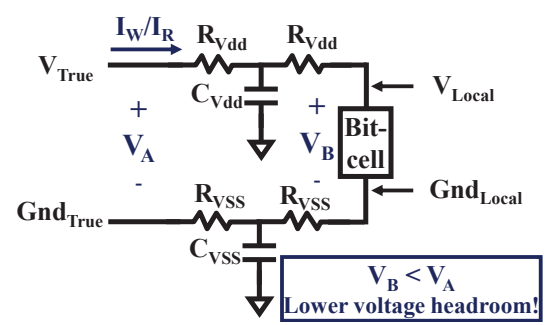

Figure 1: Voltage droop and ground bounce.

(b) Local ground bounce: The true ground $\left(\mathrm{V}_{\mathrm{SS}}\right)$ is routed on upper metal layer (distributed in metal $\mathrm{M}_{8}$ ) and connects to the transistors in $M_{1}$ (similar to $V_{d d}$ routing) (Fig. 1). Therefore, the voltage of local ground rail bounces when the charge (due to high write/read current) is dumped to it.

The magnitude of total supply noise (i.e. droop and bounce) depends on the present state of the memory bit as well as the new data being written since write current for $0 \rightarrow 0,0 \rightarrow 1,1 \rightarrow 0$ and $1 \rightarrow 1$ are different (for write), and on the stored data (for read). In this work, we leverage the supply noise generated by the write operation of emerging NVMs to launch information leakage attack. The basic methodology of the attack is given below:

Information Leakage Attack (Fig. 2a): Victim writes sensitive data pattern creating supply noise which propagates to the adversary's memory space. Adversary can exploit the faster read latency and launch several reads (with carefully chosen data) in his memory space while the victim is writing sensitive data. Adversary reads a known data (i.e. known supply noise) which adds up to the propagated noise and creates a read failure. From the read failure, adversary can detect the amount of sensitive noise generated by the victim's write operation and back calculate the Hamming Weight (HW) of the victim's sensitive write data. For example, if the victim writes a data which generates $>150 \mathrm{mV}$ droop in adversary's memory space, the adversary can conclude that victim's write data HW is $>66.77 \%$ (with some assumptions).

We have considered 1T1R RRAM-based LLC in this work as a test case. Fig. 3 shows an overview of 4MB 1T1R-based LLC. However, the attack is also applicable to other NVMs, such as STTRAM. It is notable that RRAM is usually considered for main memory owing to poor endurance. However, high endurance $\left(\sim 10^{12}\right)$ RRAM has been also proposed recently [17], which can be suitable for LLC.

Attack model and assumptions: In this work, we have assumed the followings: (i) NVM LLC is being shared by two users and the users are an adversary and a victim. (ii) bank-level parallelism (i.e., parallel reads/writes on independent banks) is employed in LLC to achieve high bandwidth; (iii) adversary has the knowledge of the amount of supply noise that can be generated by a $\mathrm{read} / \mathrm{write}$ data pattern during read/write operation initiated by him; (iv) adversary knows the propagation model of the generated supply noise (which decays with distance) and the impact of the propagated noise on the victim's read/write operation; (v) adversary is an expert in computer architecture and can exploit knobs, such as accessing specific data patterns in different predefined physical locations to prevent their replacement by policies, such as Least Recently Used (LRU).

The long write latency of emerging NVMs worsens the supply noise issue due to bank-level parallelism. Furthermore, the nature of

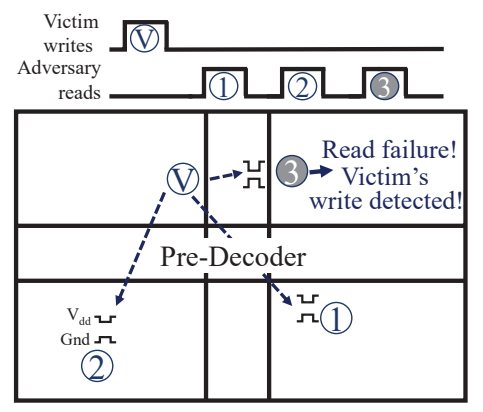

(a) Information Leakage Attack

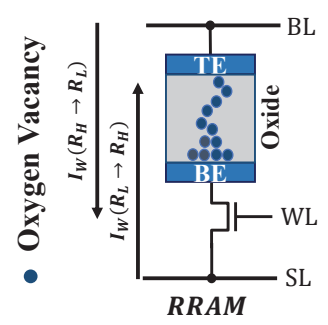

(b) RRAM Bitcell
Figure 2: (a) Information leakage attack; and, (b) schematic of RRAM bitcell.

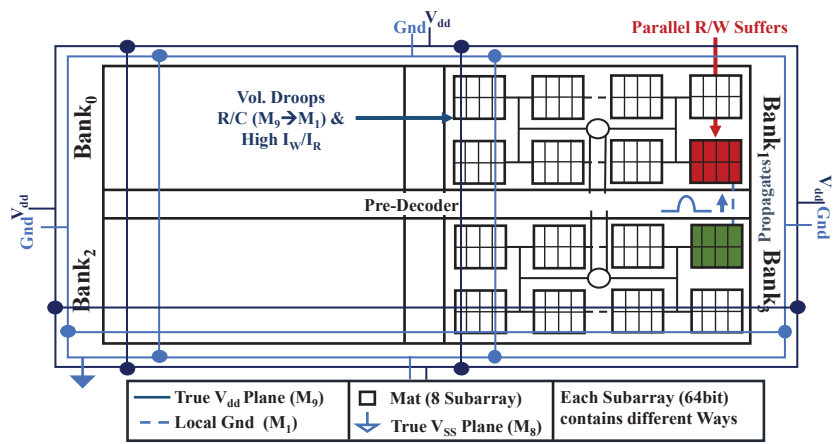

Emerging NVM LLC (4MB)

Figure 3: 1T1R-based 4MB LLC (containing 4 banks) showing supply noise. Each bank contains 8 Mats and each Mat contains 8 subarrays each producing 64bits. Each subarray has 8 Ways. Parallel read/write in Bank 1 (red) suffers due to propagation of noise from $\mathrm{Bank}_{3}$ (green) (or vice versa).

cache (for example, set associativity) and the replacement policies (for example, LRU) can force adversary's address space physically close to the victim's address space making the task easier for the adversary. A detailed approach to manipulate addresses using cache associativity and replacement policy is beyond the scope of this paper.

It should be noted that read/write operation can be affected due to both self-inflicted and parallel read/write-inflicted supply noise. Therefore, bits are tested, and optimal $\mathrm{V}_{\mathrm{dd}} / \mathrm{T}_{\text {clock }}$ are selected for successful read/write operation. However, traditional test approach may fail to validate memory functionality for all possible corner cases. Therefore, adversary's read operation can be affected by victim's parallel write operation. The adversary can leverage this to detect HW of victim's write data (details provided in Section III).

Following contributions are made in this paper: We (i) show that supply noise generated by a write operation in one bank can worsen the sense margin if a read is performed in parallel in other independent banks. Therefore, those bits can suffer from read failures; (ii) find the magnitude of supply noise required to cause a read failure; (iii) show that adversary can detect the HW of victim's write data by detecting read failures in his own address space; (iv) discuss potential design-level countermeasures.

Rest of the paper is organized as follows: Section II presents the background on RRAM and supply noise modeling and their impact on parallel read/write operation; Section III describe information 


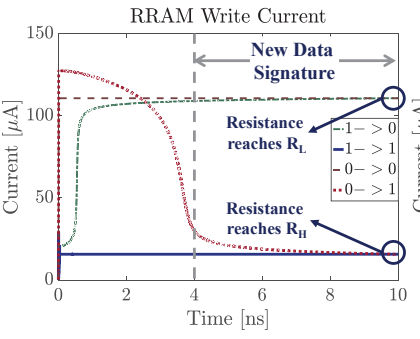

(a) Write current

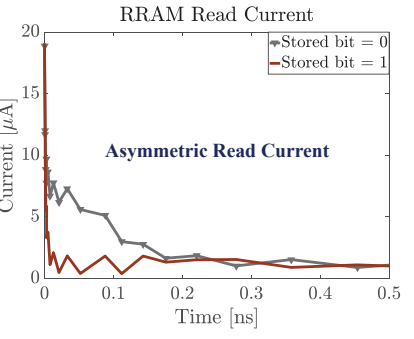

(b) Read current
Figure 4: RRAM (a) write current; and (b) read current.

leakage attacks; Section IV presents discussion on the practicality of the proposed attacks and design level mitigation techniques; Section V draws conclusion.

\section{BACKGROUND}

\subsection{Basics of RRAM}

RRAM contains an oxide material between Top/Bottom Electrode (TE/BE) (Fig. 2b). RRAM resistive switching is due to oxide breakdown and re-oxidation which modifies a Conduction Filament (CF). Conduction through the $\mathrm{CF}$ is primarily due to the transportation of electrons in the oxygen vacancies. These vacancies are created under the influence of electric field due to the applied voltage. The two states of the RRAM are termed as Low Resistance State (LRS), $\mathrm{R}_{\mathrm{L}}$ and High Resistance State (HRS), $\mathrm{R}_{\mathrm{H}}$. The process of switching the state to LRS (HRS) is known as SET (RESET). We have used ASU RRAM Verilog-A model [18] along with $65 \mathrm{~nm}$ nMOS as the access transistor for simulation and analysis. The RRAM is bipolar $\mathrm{HfO}_{\mathrm{x}^{-}}$ based resistive switching memory [18]. All the model parameters used in this work are shown in Table 1.

Asymmetric read/write current and short read latency: The total write current for writing a full cache line is a function of data pattern due to asymmetric write current (Fig. 4a) [13]. An adversary can select the write data pattern to precisely control and generate supply noise. Similarly, NVM read current is also asymmetric (Fig. 4b). Furthermore, NVM read latency can be optimized to less than a ns (Fig. 4b, 0.5ns in this work). Therefore, adversary can initiate many reads between one write operation of the victim, and detect the HW of the victim's write data (details in Section III).

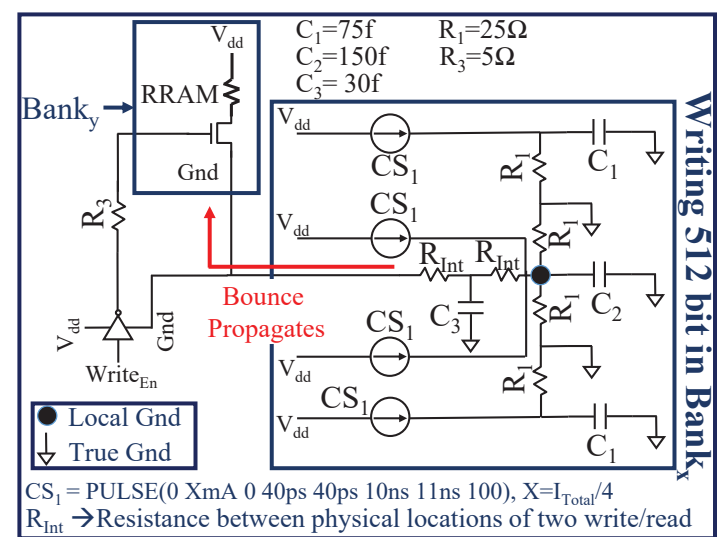

Figure 5: Equivalent circuit for modeling ground bounce.
Table 1: Parameters Used for the Simulation

\begin{tabular}{|l|r|}
\hline Parameter & Value \\
\hline \hline Access Transistor $\mathrm{W} / \mathrm{L} / \mathrm{V}_{\mathrm{T}}$ & $195 \mathrm{~nm} / 65 \mathrm{~nm} / 0.423 \mathrm{~V}$ \\
\hline RRAM Gap for $\mathrm{R}_{\mathrm{L}} / \mathrm{R}_{\mathrm{H}}$ & $0.53 \mathrm{~nm} / 1.368 \mathrm{~nm}$ \\
\hline Unit Cell Size & $12 \mathrm{~F}^{2}$ \\
\hline System Clock Frequency/V & $2 \mathrm{GHz} / 2.2 \mathrm{~V}$ \\
\hline Read/Write Latency & $0.5 \mathrm{~ns}(1$ cycle $) / 10 \mathrm{~ns}(20$ cycle $)$ \\
\hline
\end{tabular}

Table 2: Parameters used for Ground Bounce Modeling

\begin{tabular}{|l|r|}
\hline Parameter & Value \\
\hline \hline Resistance $(\Omega / \mu \mathrm{m})$ & $0.91 / 0.41 / 0.41 / 0.41 / 0.41 /$ \\
$\mathrm{M}_{1} / \mathrm{M}_{2} / \mathrm{M}_{3} / \mathrm{M}_{4} / \mathrm{M}_{5} / \mathrm{M}_{6} / \mathrm{M}_{7} / \mathrm{M}_{8}$ & $0.41 / 0.04 / 0.04[19,20]$ \\
\hline Capacitance $(\mathrm{fF} / \mu \mathrm{m})$ & $0.13 / 0.17 / 0.17 / 0.17 /$ \\
$\mathrm{M}_{1} / \mathrm{M}_{2} / \mathrm{M}_{3} / \mathrm{M}_{4} / \mathrm{M}_{5} / \mathrm{M}_{6} / \mathrm{M}_{7} / \mathrm{M}_{8}$ & $0.17 / 0.17 / 0.19 / 0.19[19,20]$ \\
\hline Miller Coupling Factor $(\mathrm{MCF})$ & 1.5 \\
\hline Via Resistance $(\Omega) \mathrm{M}_{1-2} /$ & $6 / 5 / 5 / 3 / 3 / 1 / 1(\mathrm{CVD}$ \\
$\mathrm{M}_{2-3} / \mathrm{M}_{3-4} / \mathrm{M}_{4-5} / \mathrm{M}_{5-6} / \mathrm{M}_{6-7} / \mathrm{M}_{7-8}$ & Tungsten-based) [21] \\
\hline Di-electric Constant for Cap. & $2.2 / 2.79[20]$ \\
Calculation $\left(\mathrm{C}_{\text {plate }} / \mathrm{C}_{\text {side }}\right)$ & \\
\hline Res. between $\mathrm{M}_{1}$ to Source/ & $\sim 5[22]$ \\
Drain Contact, $\mathrm{R}_{\text {Contact }}(\Omega)$ & \\
\hline
\end{tabular}

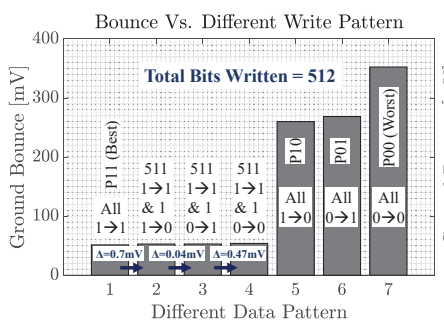

(a)

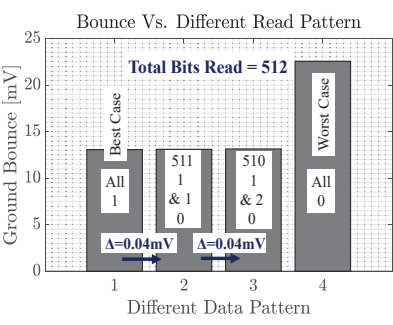

(b)
Figure 6: (a) Local ground bounce vs write data pattern; and (b) local ground bounce vs read data pattern.

\subsection{Supply Noise Modeling}

Fig. 3 shows 4MB 1T1R 4-way set associated LLC organization. All the Ways of each Mat are accessed simultaneously and buffered at the edge of each Mat, resulting in total of 512bit accesses. The figure also shows the upper layer metal plan. $V_{d d}$ plane is in $M_{9}$ and $V_{S S}$ plane is in $M_{8}$. Both $V_{d d}$ and $V_{S S}$ is implemented from $M_{7}$ to $M_{1}$ where $M_{7}, M_{5}, M_{3}$ and $M_{1}$ are horizontal and $M_{6}, M_{4}$, $M_{2}$ are vertical. The total chip area is $4970 \lambda \times 3950 \lambda$ where each bank occupies $2046 \lambda \times 1536 \lambda$ and the remaining is occupied by the peripheral circuitry. Note that $\lambda$ is the feature size.

Ground bounce: Fig. 5 shows the circuit used for ground bounce modeling. We modeled the resistance of path $M_{1}$ to $M_{8}$ by $R_{1}$ using $65 \mathrm{~nm}$ layout parameters (Table II) [19, 20]. Each metal layer R/C and via resistance between metal layers are also given in Table II. Our estimation shows that $\mathrm{R} 1$ is equivalent to $\sim 25 \Omega$ [16]. The magnitude of ground bounce depends on this value. Capacitance calculation is omitted for brevity. We also modeled the resistance $\mathrm{R}_{\text {int }}$ which represents the equivalent resistance between the local ground of one address of one bank to the local ground of another address of another bank. Our estimation shows that the lowest (closest two addresses of two banks) $R_{\text {int }}$ is $1.63 \Omega$. Average read/write current 


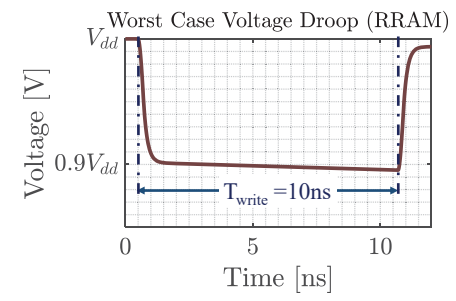

(a)

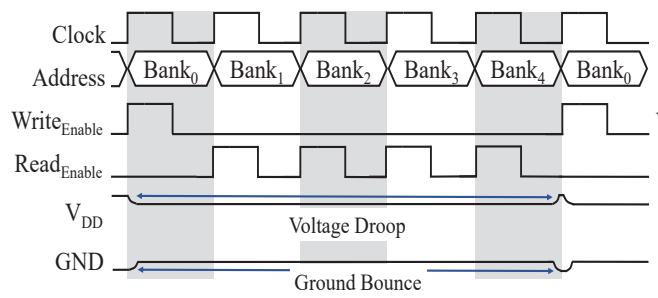

(b)

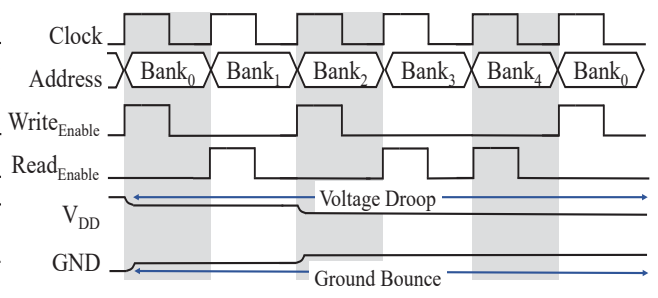

(c)

Figure 7: Worst-case (P00) voltage droop when writing all the bits to one Mat of Bank 3 (Fig. 3); (b) four reads are initiated between two writes. We call it $1 \mathrm{X}$ write; and (c) three reads and one write are initiated between two writes. We call it $2 \mathrm{X}$ write.

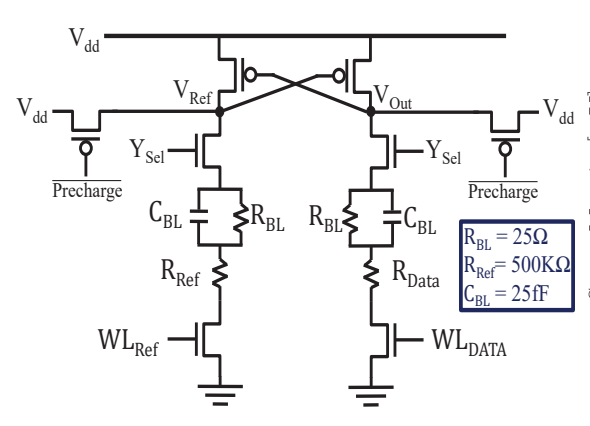

(a)

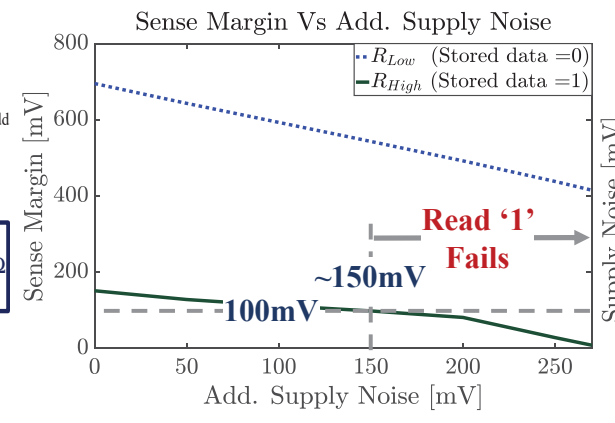

(b)

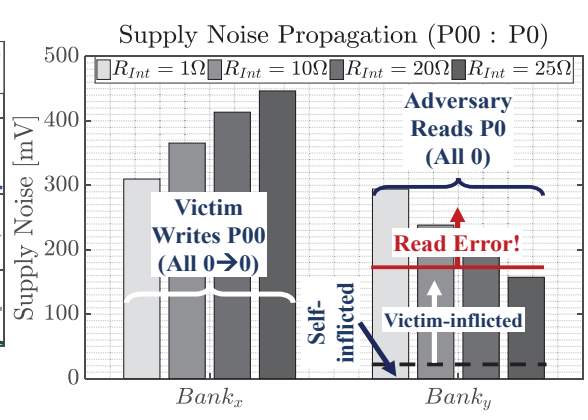

(c)

Figure 8: (a) Single-ended read circuitry used it this work (circuit redrawn from US patent no. US7515461B2) [23]; (b) read failure for data ' 1 ' is observed above $150 \mathrm{mV}$ of additional supply noise; and (c) victim writes and adversary reads P00/P0 in Bank $_{x} /$ Bank $_{y}$ respectively. Victim's write can be detected with adversary's read failure if victim is writing very close and generating high supply noise.

for a full cache line is divided into four constant Current Sources (CSs). Therefore, current magnitude of $\mathrm{CS}, \mathrm{XmA}$ is equal to $\mathrm{I}_{\mathrm{Total}} / 4$ (for example, $\mathrm{I}_{\text {Total }}=56.32 \mathrm{~mA}$ and $\mathrm{X}=14.08$ for $512 \mathrm{bits}$ of $0 \rightarrow 0$ writing) and each one presents total read/write current for $128 \mathrm{bits}$.

Fig. 6a shows the bounce generated by a full cache line write for various write data patterns. It is notable that $1 \rightarrow 1$ (we call it P11) write creates lowest $(\sim 51.42 \mathrm{mV})$ (best-case), and $0 \rightarrow 0$ (we call it P00) creates highest $(\sim 352.46 \mathrm{mV})$ (worst-case) ground bounce. It is also evident that other data patterns create a bounce in between P11 and P00. Furthermore, the bounce can be controlled at the granularity of $1 \mathrm{mV}$ by choosing corresponding write data pattern. Fig. 6b shows the ground bounce generated by various read data patterns. Highest/lowest bounce generated by reading a data is $\sim 22.5 \mathrm{mV}$ (when all 512bits are 1s, we call it $\mathrm{P} 1$ ) $/ \sim 13.1 \mathrm{mV}$ (when all 512bits are 0 s, we call it $\mathrm{P} 0$ ). The adversary can control the generated bounce by reading specific data patterns and store these patterns in his memory space before launching the attack.

Voltage droop: Droop is modeled using a circuit model similar to Fig. 5 (details omitted for brevity). Simulation indicates that supply voltage can droop to $\sim 0.9 \mathrm{~V}_{\mathrm{dd}}$ when writing $\mathrm{P} 00$ to all the bits of a Mat of Bank 3 (Fig. 7a).

In the rest of the paper, we have combined the magnitude of droop and bounce and call it supply noise. It should be noted that supply noise can be both, self-inflicted and parallel-read/writeinflicted. We call the parallel-read/write-inflicted supply noise as the additional supply noise. We have ignored the droop caused by the read operation (due to insignificant magnitude) and considered only the ground bounce. Therefore, supply noise generated by the read operation is effectively ground bounce only. However, both droop and bounce component of supply noise are considered for a write operation.

\subsection{Parallel Read/Write Operation}

RRAM write requires multiple clock cycles. For example, the required number of clock cycles are 5 (1) with a system clock frequency of $2 \mathrm{GHz}$ and write (read) latency of $2.5 \mathrm{~ns}(0.5 \mathrm{~ns})$. However, the throughput will degrade if memory access is completely stopped during 5 or 1 cycles (Fig. 7b-7c). In practice, RRAM write latency is even higher (10ns, 20cycles for this work). Therefore, parallelism is used to perform write/read in successive cycles (can be initiated by different users). The parallel access can take the following forms:

1X write: Read can be initiated in the next 4 cycles in other banks (Fig. 7b) for adversary when a write has been initiated for the victim. These data are processed in the pipeline to maintain high throughput. Read operations initiated in cycles 2, 3, 4 and 5 will experience failure (due to supply noise propagation to those banks) owing to, (a) poor sense margin at lower voltage headroom; (b) higher access transistor resistance at lower word-line voltage. We call this write/read scheme $1 \mathrm{X}$ write.

nX write: Multiple (n) writes can be initiated with multiple reads. For example, one write along with 3 reads can be initiated in the next four clock cycles in other banks (Fig. 7c) for adversary when a write has been initiated for the victim $(n=2)$. The second write will draw additional current from the supply which might add to the existing supply noise. Furthermore, the second write might fail due to the noise propagated from the first write operation (or vice-versa). We call this write/read scheme $2 \mathrm{X}$ write. 


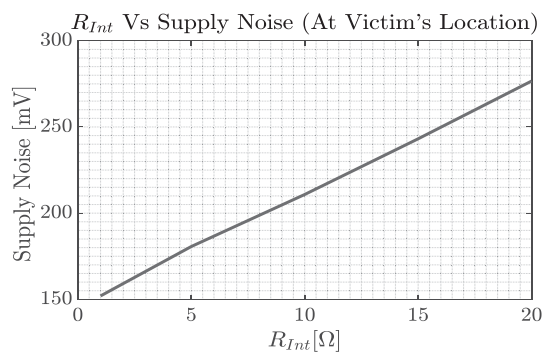

(a)

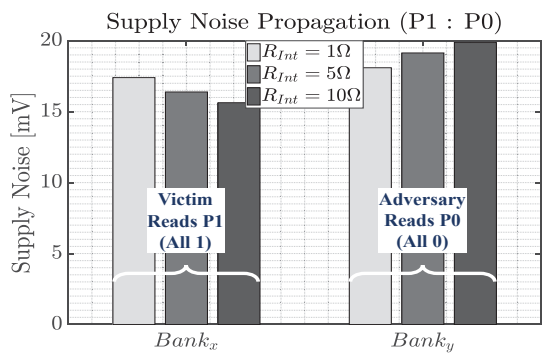

(b)

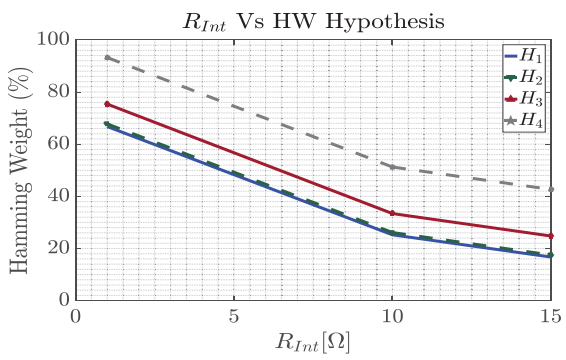

(c)

Figure 9: (a) RInt vs noise generated from victim's writing at victim's location; (b) both victim/adversary read P1/P0 in Bank $_{\mathbf{x}} /$ Bank $_{\mathbf{y}}$ respectively; and (c) $\mathrm{R}_{\text {Int }}$ boundary values for all four hypotheses.

\section{INFORMATION LEAKAGE ATTACK}

\subsection{Detection of victim's write initiation:}

The adversary needs to know when and where (physical location) the victim is writing in order to launch information leakage attacks effectively. One possible approach adopted by the adversary is to store data that generates highest supply noise (i.e., all 0 s in the stored data) in various locations of the memory and read them frequently. If a read error occurs, it can be assumed that the victim has initiated a write, physically close to adversary's read location which caused the read failure. This is true since victim's read cannot generate enough noise to cause failure to adversary's parallel read operation. The adversary can keep reading different addresses to detect victim's write operation as read latency is significantly shorter than the write latency. However, detection of victim's write operation by observing adversary's write failure is not feasible because of long write latency.

Fig. 8a presents the single-ended read circuity [23] used in this work. The read circuity is proposed in [23]. We have considered $\mathrm{R}_{\mathrm{BL}}$ $=25 \Omega, \mathrm{R}_{\mathrm{Ref}}=500 \mathrm{~K} \Omega$ and $\mathrm{C}_{\mathrm{BL}}=25 \mathrm{fF}$ for read operation analysis. We further analyze the sense margin for both data ' 0 ' and ' 1 ' using the read circuitry of Fig. 8a. Fig. 8b shows that sense margin reduces with additional supply noise. However, ground bounce affects sense margin more compared to droop as it, i) reduces the discharge current, and ii) reduces $\mathrm{V}_{\mathrm{GS}}$ of access transistor (i.e., $\mathrm{R}_{\text {Transistor }}$ is higher) while voltage droop only reduces the discharge current. It is evident from Fig. 8b that if victim's write generates supply noise in a way that the adversary incurs additional supply noise > $150 \mathrm{mV}$, adversary will read data ' 1 ' incorrectly. Therefore, adversary can detect victim's write operation by observing read failure and further approximate HW of victim's write data (explained in next subsection). Fig. 8c shows that victim writes and adversary reads $\mathrm{P} 00 / \mathrm{P} 0$ in $\mathrm{Bank}_{\mathrm{x}} / \mathrm{Bank}_{\mathrm{y}}$ respectively. Victim's write can be detected by observing adversary's read failure if $\mathrm{R}_{\text {Int }}$ between victim and adversary is $<23 \Omega$ and victim is writing P00 (generating worst-case supply noise). Fig. 9a shows the required supply noise generated at victim's location with $\mathrm{R}_{\mathrm{Int}}$ in order to be detected at adversary's read location (i.e. trigger read error).

Fig. 9b shows that both victim and adversary read P1/P0 in $\mathrm{Bank}_{\mathrm{x}} / \mathrm{Bank}_{\mathrm{y}}$ respectively. The adversary does not incur read error due to noise generated by victim's read. Therefore, victim's read cannot be detected by adversary's read (even if victim reads $\mathrm{P} 0$ ) and the adversary cannot approximate HW of victim's read data.

\subsection{Data Information Approximation}

In [16], it has been shown that if a write operation incurs an additional supply noise of more than $120 \mathrm{mV}$, both $1 \rightarrow 0$ and $0 \rightarrow 1$ write operation fails for the similar RRAM employed in this work. However, if the write operation incurs $>50 \mathrm{mV}$ but $<120 \mathrm{mV}$ of supply noise, $0 \rightarrow 1$ write fails but $1 \rightarrow 0$ write succeeds. Furthermore, if the write operation incurs $<50 \mathrm{mV}$ of supply noise, both $1 \rightarrow 0$ and $0 \rightarrow 1$ write successfully. These observations can be leveraged by adversary to detect HW of victim's write data.

RRAM write waveforms (Fig. 4a) show that it can be divided into two regions with respect to time. The first $4 \mathrm{~ns}$ and last $6 \mathrm{~ns}$ represents old and new data of the memory cell respectively. Write current in the last region is approximately two values: one for data ' 1 ' (low current) and one for data ' 0 ' (very high current). Adversary can leverage these observations to approximate the HW of the victim's write data. Therefore, adversary's widow of interest (WOI) will be the last region once the write initiation is detected. Adversary can extract information by read/write operation (during WOI) to near-by addresses (with known content) where the read failure is first observed (write initiation detection) in the following ways:

i) Hypothesis $\mathbf{1}\left(\mathbf{H}_{\mathbf{1}}\right)$ : Adversary reads P1 (lowest noise by adversary). If read fails, it can be concluded that victim is adding $>150 \mathrm{mV}$ of supply noise to adversary's read location. Therefore, $\mathrm{HW}$ of victim's write data must be $>66.77 \%$ (for $\mathrm{R}_{\text {Int }}=1 \Omega$ ).

ii) Hypothesis $2\left(\mathbf{H}_{2}\right)$ : Next, adversary reads $\mathrm{P} 0$ (highest noise by adversary). If read does not fail, it can be concluded that HW of victim's write data must be $<67.76 \%$ (for $\mathrm{R}_{\text {Int }}=1 \Omega$ ). Else, $66.77 \%<\mathrm{H}$ $\mathrm{W}<67.76 \%$.

Following hypotheses can be made from the write failure characteristics observed by the adversary in the remaining $5 \mathrm{~ns}$ of WOI:

iii) Hypothesis $\mathbf{3}\left(\mathbf{H}_{3}\right)$ : Adversary initiates a write (that has both $1 \rightarrow 0$ and $0 \rightarrow 1$ switching) and reads the written data. If adversary incurs both $0 \rightarrow 1$ and $1 \rightarrow 0$ write failures, it can be concluded that victim is adding $>120 \mathrm{mV}$ but $<150 \mathrm{mV}$ of supply noise. Therefore, $67.76 \%<\mathrm{HW}<75.37 \%$ (for $\mathrm{R}_{\text {Int }}=1 \Omega$ ).

iv) Hypothesis $4\left(\mathbf{H}_{\mathbf{4}}\right)$ : If only $0 \rightarrow 1$ fault occurs, then victim is adding $>50 \mathrm{mV}$ but $<120 \mathrm{mV}$ of supply noise. Therefore, $75.37 \%<\mathrm{HW}$ $<93.23 \%$ (for $\mathrm{R}_{\text {Int }}=1 \Omega$ ).

All the above boundary conditions for the hypotheses could be calculated by adversary by assuming $\mathrm{R}_{\text {Int }}=1 \Omega$. In practice, the adversary does not know victim's exact write location. The boundary conditions can be different (Fig. 9c) depending on the distance (i.e. 
$\mathrm{R}_{\text {Int }}=1 \Omega$ ). However, this information can be used in other attack models, such as SCA, to reduce the search space significantly and thereby improving the attack.

This study is carried out for RRAM LLC with a specific power grid design. However, we believe that the conclusions drawn in this work are applicable to broad range of NVMs and power grids. A better grid or NVM with scaled read/write current could reduce the supply noise but may not eliminate the challenge completely.

\section{DISCUSSION}

\subsection{Attack Countermeasures}

Following techniques can prevent or alleviate the attacks: (i) Sequential read/write access: This can be a naïve solution as non-pipelined access hurts system throughput. However, adversary can not detect victim's write initiation or HW of write data; (ii) Intelligent architecture: Parallel operations of different processes can be initiated to addresses with highest possible $\mathrm{R}_{\text {Int }}$. This will alleviate the issue to some extent; (iii) Good quality power/ground grid: A good power/ground grid reduces $\mathrm{R}_{1}$ (in Fig. 5) which in turn reduces supply noise. However, this cannot eliminate the issue completely; (iv) Power rail separation for each bank: Separation of $V_{d d}$ and $V_{S S}$ rails between parallel accessed banks will prevent propagation of supply noise from one bank to another. However, this will incur significant area-overheard and reduce the power rail capacitance (which is not desirable).

\subsection{Memory Testing \& Attack Effectiveness}

The proposed attacks can be prevented if exhaustive testing of the memory is performed considering all worst-case patterns, all worstcase possible location combinations, all possible parallel read/write conditions (performed under bit-to-bit process variations and wide range of temperatures $\left(-100^{\circ} \mathrm{C}\right.$ to $200^{\circ} \mathrm{C}$ for example) $)$ and optimal $\mathrm{V}_{\mathrm{dd}} / \mathrm{T}_{\text {Clock }}$ is chosen accordingly. On one hand, exhaustive testing of the memory with fully integrated system is impractical as it: i) increases test time and time to market unacceptably. A rough estimation shows that the test time can be extended to $27.06 \mathrm{~s}$ per chip! [16] This is unacceptable as the total test time (including all other tests) of a chip is typically around 2-3sec (unit test time $=2.7 \mathrm{sec}$ shown in [24]); ii) guard-bands system performance for situations that may never arise under normal workloads (especially true for high-performance applications).

\section{CONCLUSION}

In this work, we show that adversary can detect victim's write initiation by observing read failure in his memory space. Furthermore, adversary can approximate HW of victim's write data which can be further utilized to strengthen other attack models, such as SCA.

\section{ACKNOWLEDGEMENT}

This work is supported by SRC (2727.001), NSF (CNS- 1722557, CCF-1718474 and DGE-1723687) and DARPA Young Faculty Award (D15AP00089).

\section{REFERENCES}

[1] A. Nigam, C. W. Smullen, V. Mohan, E. Chen, S. Gurumurthi, and M. R. Stan, "Delivering on the promise of universal memory for spin-transfer torque ram
(STT-RAM)," in IEEE/ACM International Symposium on Low Power Electronics and Design, pp. 121-126, Aug 2011.

[2] D. C. Worledge, G. Hu, P. L. Trouilloud, D. W. Abraham, S. Brown, M. C. Gaidis, J. Nowak, E. J. O’Sullivan, R. P. Robertazzi, J. Z. Sun, and W. J. Gallagher, "Switching distributions and write reliability of perpendicular spin torque MRAM," in 2010 International Electron Devices Meeting, pp. 12.5.1-12.5.4, Dec 2010.

[3] Y. Wu, S. Yu, X. Guan, and H. S. P. Wong, "Recent progress of resistive switching random access memory (RRAM)," in 2012 IEEE Silicon Nanoelectronics Workshop (SNW), pp. 1-4, June 2012

[4] A. Pirovano, A. L. Lacaita, F. Pellizzer, S. A. Kostylev, A. Benvenuti, and R. Bez, "Low-field amorphous state resistance and threshold voltage drift in chalcogenide materials," IEEE Transactions on Electron Devices, vol. 51, pp. 714-719, May 2004.

[5] Y. M. Kang and S. Y. Lee, "The challenges and directions for the mass-production of highly-reliable, high-density 1T1C FRAM," in 2008 17th IEEE International Symposium on the Applications of Ferroelectrics, vol. 1, pp. 1-2, Feb 2008.

[6] A. Chen, "A review of emerging non-volatile memory (nvm) technologies and applications," Solid-State Electronics, vol. 125, pp. 25 - 38, 2016. Extended papers selected from ESSDERC 2015.

[7] C. J. Xue, G. Sun, Y. Zhang, J. J. Yang, Y. Chen, and H. Li, "Emerging nonvolatile memories: Opportunities and challenges," in 2011 Proceedings of the Ninth IEEE/ACM/IFIP International Conference on Hardware/Software Codesign and System Synthesis (CODES+ISSS), pp. 325-334, Oct 2011.

[8] A. De, M. N. I. Khan, J. Park, and S. Ghosh, "Replacing eflash with sttram in iots: Security challenges and solutions," fournal of Hardware and Systems Security, vol. 1, pp. 328-339, Dec 2017.

[9] "Intel Optane Memory Series." https://ark.intel.com/products/97544/ Intel-Optane-Memory-Series-16GB-M_2-80mm-PCIe-3_0-20nm-3D-Xpoint, 2015. [Online; accessed May-03-2018].

[10] S. Motaman, M. N. I. Khan, and S. Ghosh, "Novel application of spintronics in computing, sensing, storage and cybersecurity," in 2018 Design, Automation Test in Europe Conference Exhibition (DATE), pp. 125-130, March 2018.

[11] J.-W. Jang, J. Park, S. Ghosh, and S. Bhunia, "Self-correcting STTRAM under magnetic field attacks," in Proceedings of the 52Nd Annual Design Automation Conference, DAC '15, (New York, NY, USA), pp. 77:1-77:6, ACM, 2015.

[12] S. Ghosh, M. N. I. Khan, A. De, and J. W. Jang, "Security and privacy threats to on-chip Non-Volatile Memories and countermeasures," in 2016 IEEE/ACM International Conference on Computer-Aided Design (ICCAD), pp. 1-6, Nov 2016.

[13] K. Shamsi and Y. Jin, "Security of emerging non-volatile memories: Attacks and defenses," in 2016 IEEE 34th VLSI Test Symposium (VTS), pp. 1-4, April 2016.

[14] M. N. I. Khan, S. Bhasin, A. Yuan, A. Chattopadhyay, and S. Ghosh, "Side-channel attack on STTRAM based cache for cryptographic application," in 2017 IEEE International Conference on Computer Design (ICCD), pp. 33-40, Nov 2017.

[15] R. K. Aluru and S. Ghosh, "Droop mitigating last level cache architecture for STTRAM," in Proceedings of the Conference on Design, Automation \& Test in Europe, DATE '17, (3001 Leuven, Belgium, Belgium), pp. 262-265, European Design and Automation Association, 2017

[16] M. N. I. Khan and S. Ghosh, "Fault injection attacks on emerging non-volatile memory and countermeasures," in Proceedings of the 7th International Workshop on Hardware and Architectural Support for Security and Privacy, HASP '18, (New York, NY, USA), pp. 10:1-10:8, ACM, 2018.

[17] M.-J. Lee, C. B. Lee, D. Lee, S. R. Lee, M. Chang, J. H. Hur, Y.-B. Kim, C.-J. Kim, D. H. Seo, S. Seo, U.-I. Chung, I.-K. Yoo, and K. Kim, "A fast, high-endurance and scalable non-volatile memory device made from asymmetric $\mathrm{Ta}_{2} \mathrm{O}_{5-\mathrm{x}} / \mathrm{TaO}_{2-\mathrm{x}}$ bilayer structures," Nature Materials, vol. 10, pp. 625 EP -, Jul 2011. Article.

[18] P. Y. Chen and S. Yu, "Compact modeling of RRAM devices and its applications in 1T1R and 1S1R array design," IEEE Transactions on Electron Devices, vol. 62, pp. 4022-4028, Dec 2015.

[19] "Interconnect: Capacitance and Resistance for $65 \mathrm{~nm}$ technology." http://ptm.asu. edu/, 2005. [Online; accessed May-03-2018].

[20] "Wire Capacitance and Resistance Calculator for 65nm." http://users.ece.utexas. edu/ mcdermot/vlsi-2/Wire_Capacitance_and_Resistance_65nm.xls, 2008. [Online; accessed May-03-2018].

[21] I. Shao, J. M. Cotte, B. Haran, A. W. Topol, E. E. Simonyi, C. Cabral, and H. Deligian$\mathrm{ni}$, "An alternative low resistance MOL technology with electroplated rhodium as contact plugs for 32nm CMOS and beyond," in 2007 IEEE International Interconnect Technology Conferencee, pp. 102-104, June 2007.

[22] X. Li, W. Zhao, Y. Cao, Z. Zhu, J. Song, D. Bang, C. C. Wang, S. H. Kang, J. Wang, M. Nowak, and N. Yu, "Pathfinding for 22nm CMOS designs using Predictive Technology Models," in 2009 IEEE Custom Integrated Circuits Conference, pp. 227230, Sept 2009.

[23] T. D. Happ, H. L. Lung, and T. Nirschl, "Current compliant sensing architecture for multilevel phase change memory,” 2009. Patent No. US7515461B2, Filed January 5th, 2007, Issued April 7th., 2009.

[24] "Addressing Test Time Challenges," 2017. 